\title{
Amérique Centrale. Défis et urgences de l'éducation en Amérique Centrale
}

Le point sur...

\section{Luis Muelle}

\section{(2) OpenEdition}

\section{Journals}

Édition électronique

URL : http://journals.openedition.org/ries/1413

DOI : $10.4000 /$ ries. 1413

ISSN : 2261-4265

\section{Éditeur}

Centre international d'études pédagogiques

Édition imprimée

Date de publication : 1 septembre 2004

Pagination : 15-17

ISBN : 978-2-85420-561-8

ISSN : 1254-4590

\section{Référence électronique}

Luis Muelle, «Amérique Centrale. Défis et urgences de l'éducation en Amérique Centrale », Revue internationale d'éducation de Sèvres [En ligne], 36 | septembre 2004, mis en ligne le 18 novembre 2011 consulté le 24 avril 2019. URL : http://journals.openedition.org/ries/1413 ; DOI : 10.4000/ries.1413

Ce document a été généré automatiquement le 24 avril 2019

(c) Tous droits réservés 


\title{
Amérique Centrale. Défis et urgences de l'éducation en Amérique Centrale
}

\author{
Le point sur...
}

\author{
Luis Muelle
}

1 En 2000, le rapport Demain est trop tard ${ }^{1}$ avait signalé l'alarmant retard accumulé par les pays d'Amérique Centrale pour relever leurs défis éducatifs : une gestion centralisée inefficiente et peu favorable aux innovations; un investissement insuffisant et inéquitable ; la dégradation du métier d'enseignant, conséquence, en partie, du niveau des salaires; et enfin, l'absence de normes d'évaluation pour mesurer les résultats des élèves.

2 Pour y faire face, un certain nombre d'actions possibles avaient été suggérées : transférer la responsabilité de la gestion de l'école aux parents d'élèves, aux enseignants et à la communauté en général ; orienter l'investissement vers l'enseignement préscolaire et secondaire ; valoriser la profession enseignante à travers une politique juste de salaires ; améliorer la qualité de la formation initiale et continue des enseignants; enfin, établir des normes d'apprentissage communes pour les élèves.

3 Malgré les différentes initiatives entreprises dans ces domaines, les résultats s'avèrent encore insuffisants. La lenteur des améliorations risque de faire reculer l'éducation dans la région et d'obérer l'avenir. La préoccupation et le débat sont encore très présents chez de nombreux responsables politiques centraméricains.

4 Les résultats des apprentissages des élèves montrent un niveau modeste. Les résultats obtenus dans les tests sont décourageants et posent sérieusement la question de la qualité de l'enseignement. Ainsi, au Nicaragua, moins de $8 \%$ des élèves de CE2 atteignent le niveau considéré comme "performance élevée » en espagnol et en mathématiques et ce pourcentage s'abaisse à $3 \%$ pour le Honduras.

5 Le niveau éducatif atteint par la population reste insuffisant. Malgré l'extension de la couverture scolaire dans l'enseignement de base, on constate encore l'existence de 
niveaux élevés d'analphabétisme. Dans la majorité des pays, le taux d'analphabétisme est encore supérieur à $15 \%$, chiffre qui est plus élevé que la moyenne pour l'Amérique latine. Le niveau d'éducation de la population active de plus de vingt-cinq ans atteint en moyenne moins de six années de scolarité, chiffre inférieur à la moyenne mondiale de six à sept ans et bien éloigné des dix ans pour les pays développés.

Malgré les importants efforts pour satisfaire la demande scolaire, plus de la moitié des enfants en âge préscolaire et au niveau secondaire ne fréquentent pas l'école. La scolarité primaire universelle n'est pas encore atteinte: au Nicaragua, la scolarisation se situe à $82 \%$ et à $85 \%$ au Guatemala ${ }^{2}$. Pour le secondaire, elle varie de $36 \%$ au Nicaragua à $62 \%$ à Panama. Enfin, la proportion atteint $27 \%$ (Nicaragua) et $51 \%$ (Guatemala) pour les enfants en âge préscolaire (quatre à six ans).

7 Les populations d'origine indigène connaissent de forts désavantages face aux autres groupes sociaux. Ainsi, au Guatemala, bien que cette population représente près de $40 \%$ en 2000 , sa présence atteint $24 \%$ dans l'enseignement secondaire et $13 \%$ dans le supérieur. À Panama, les enfants d'origine indigène ont une participation presque nulle au niveau universitaire. De même, les jeunes des zones rurales possèdent un niveau de scolarité inférieur à ceux des zones urbaines.

Trois années après les réformes proposées dans Demain est trop tard, constatant que les progrès accomplis sont prometteurs mais lents, la publication récente de Es Hora de actuar ${ }^{3}$ (Il est temps d'agir) est une nouvelle mise en garde. Le rapport réitère la nécessité d'améliorer d'urgence l'état actuel des systèmes éducatifs et propose de nouvelles actions pour approfondir et accélérer ces évolutions.

9 La décentralisation doit se poursuivre afin de garantir la participation effective des parents et de la communauté éducative. Elle doit s'accompagner du transfert des ressources financières aux établissements scolaires.

Un des mécanismes pour agir sur les inégalités à l'école consiste à concentrer les ressources disponibles sur les élèves les plus défavorisés afin de faciliter leur accès à l'éducation. Ceci exige de repenser les politiques actuelles de financement, tournées davantage vers l'enseignement supérieur, pour les réorienter vers le préscolaire et le secondaire.

11 Pour améliorer les motivations des enseignants, il faut mettre en œuvre une politique plus juste des salaires. L'idée actuelle d'ajuster l'avancement de carrière selon les résultats obtenus par les élèves connaît de très fortes oppositions de la part des enseignants. Cette logique exige de mettre en place un système de sanctions et de récompenses accepté et négocié avec les représentants des enseignants.

Il faut promouvoir des normes de compétences régionales. L'harmonisation des compétences à atteindre dans toutes les disciplines et degrés de l'enseignement primaire au niveau centraméricain a vu le jour sur proposition de la $\mathrm{CECC}^{4}$; les objectifs et les contenus de ces programmes commencent à s'intégrer à ceux déjà en place dans chaque pays. Une proposition concernant l'enseignement secondaire est en cours de préparation.

13 Enfin, la construction d'indicateurs sur le fonctionnement des établissements, des enseignants, des chefs d'établissements et bien sûr des élèves devient incontournable pour assurer le pilotage du système éducatif. La transparence des résultats de toute l'activité éducative est indispensable pour une éducation plus démocratique et juste.

14 L'éducation a toujours été reconnue comme un facteur décisif pour le développement humain en raison de son incidence sur la vie politique, sociale, culturelle, économique, et 
démocratique des sociétés. Aussi, le dernier sommet des chefs d'État des Amériques ${ }^{5}$ considère la lutte contre l'augmentation du taux d'analphabétisme comme une priorité immédiate. De même, il existe une forte volonté politique pour continuer à promouvoir l'accès à une éducation de base de qualité pour tous, en se fondant sur les principes de participation, d'équité, de pertinence et d'efficacité ainsi que pour encourager le processus de développement de ces pays sans discrimination ni exclusion.

\section{NOTES}

1. Mañana es muy Tarde, Comisión Centromericana para la Reforma de la Educación, PREAL, 2000.

2. UNESCO, Recueil de données mondiales sur l'éducation, Institut de statistique, Montréal, 2004.

3. Es Hora de actuar, Informe del progreso educativo en Centroamérica y la República Dominicana, PREAL, 2003.

4. Proyecto de estándares para la Educación Primaria, OEI/CECC : Coordinación Educativa y Cultural Centroamericana, San José, 2000.

5. Cumbre Extraordinaria de las Américas, Monterrey, México, janvier 2004.

\section{INDEX}

Mots-clés : système éducatif

Index géographique : Amérique centrale

\section{AUTEUR}

\section{LUIS MUELLE}

Chargé de programme, Département éducation, Centre international d'études pédagogiques. 